\title{
A Modified Watershed Segmentation Algorithm using Distances Transform for Image Segmentation
}

\author{
Pinaki Pratim Acharjya \\ Assistant Professor, Department of CSE \\ Bengal Institute of Technology and Management \\ Santiniketan, West Bengal, Pin - 731236
}

\author{
Dibyendu Ghoshal \\ Associate Professor, Department of ECE \\ National Institute of Technology \\ Agartala, Tripura, Pin - 799001
}

\begin{abstract}
In this paper, we propose a modified watershed algorithm for image segmentation using distances transform and image smoothing method, an improved version of watershed segmentation. This algorithm allows better boundary localization due to the edge information brought by watersheds. Thus, the proposed method has been found to be able to reduce over segmentation and this would ultimately lead to easier handling by the machine towards higher level of processing at subsequent stages. The algorithm has been tested on colored image obtained from real life and has been found to yield satisfactory segmentation results.
\end{abstract}

\section{Keywords}

Over segmentation, Image smoothing, Watersheds.

\section{INTRODUCTION}

The image segmentation has been as widely applied as in almost every field that is related to digital image processing and for most image understanding activities like image description and recognition [1-2], image visualization [3-4], and object based image compression [5-6]. These activities mostly depend on the results of segmentation. The role of the segmentation process is to segregate an image into regions with one or more characteristics. It is a procedure to separate an image into multiple pixels known as super pixels. Several methods and approaches are introduced into the area of segmentation [7-8], among them a well known method is watershed algorithm [9-13]. The watershed transform is a powerful morphological tool for image segmentation. Beucher and Lantuejoul were the first to apply the concept of watershed to digital image segmentation problems. A good number of works has already been carried out on watershed segmentation and these are available in the published or online literature [14-28].

Unfortunately, the watershed segmentation technique leads to an over segmentation problem [19]. Over segmentation is the process by which the image being segmented into small segments [20]. These small segments are usually uniform in size and commonly referred to as super pixels. Over segmentation through extracts important boundaries, may lead to creating insignificant boundaries.

In the present study a watershed segmentation technique using distance transform [21-22] has been carried out with the image smoothing approach., We need to use distance transforms technique for binary images (obtained from the original images) to preprocess the image to make it suitable for watershed segmentation. In a binary image, there are only two gray levels 0 and 1 standing for black and white. If two black blobs are connected together in a binary image, only one minimum and catchment basin will appear in the topographic surface.

The smoothing technique is applied on the binary image in followed by the distance transform technique to find the watershed segmented image. The image of pears has been chosen as an input image which is applied in this paper obtained from real life, and subsequently watershed segmentation has been achieved using the standard MAT LAB command. By smoothing the binary image it has been found that, the over segmentation is appreciably less in the segmented image than those obtained by without using image smoothing technique on the binary image. It has been already found that the lower scale of over segmentation will enable the computers to process the segmented images more easily and the experimental result presented in this paper is obtained by using MATLAB.

\section{OVERVIEW OF THE PROBLEM}

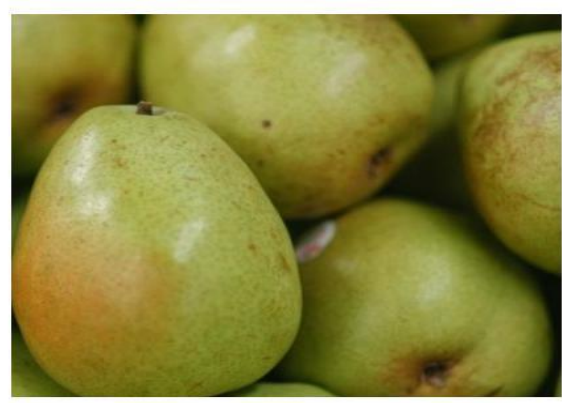

Fig. 1: Original image.

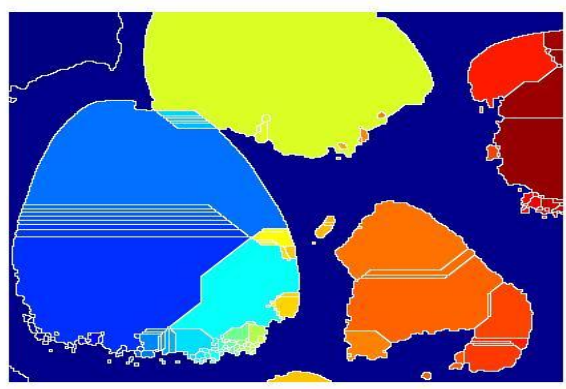

Fig. 2: Segmented image after applying of watershed segmentation using distance transform without smoothing technique where, over segmentation is clearly visible. 
When the watershed segmentation using distance transform is directly applied in to a binary image, the result of the watershed transform of the original image contains several small regions which produces an over segmented image. Figure 1, is the original image and figure 2, shows the over segmented image where we have applied the watershed segmentation using distance transform without smoothing the binary image, which makes this result hardly useful. The use of smoothing technique on the binary image is an effective solution to reduce the number of minima of the image and, thus, the number of regions.

\section{WATERSHED SEGMENTATION USING THE DISTANCE TRANSFORM}

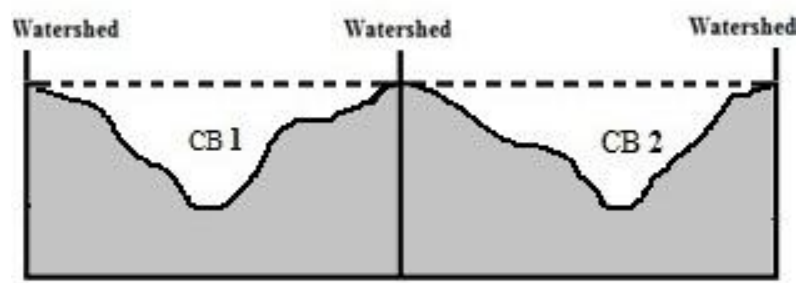

Fig. 3: Watershed segmentation-local minima of gray level yield catchment basins, local maxima define the watershed lines.

The Watershed method or the watershed transform is an image segmentation technique based on gray-scale mathematical morphology. It can be classified as a regionbased segmentation approach where the main idea behind this method comes from geography. If a landscape or topographic relief which is flooded by water, the divide lines of the domains of attraction of rain falling over the region forms watersheds. An alternative approach is to imagine the landscape being immersed in a lake, with holes pierced in local minima also called catchment basins (CB), will fill up with water starting at these local minima, and, at points where water coming from different basins would meet, dams are built. When the water level has reached the highest peak in the landscape, the process is stopped. As a result, the landscape is partitioned into regions or basins separated by dams, called watershed lines or simply watersheds

For digital image segmentation, the distance transform method is commonly used in conjunction with the watershed transform. The distance transform is the distance from every pixel to the nearest pixel of a binary image. In distance transform method every 1-valued pixel has a distance transform value of 0 because its closest nonzero pixel is itself. In below, figure 4(a) shows a binary image matrix, and in figure 4(b) shows the corresponding distance transform.

\begin{tabular}{|l|l|l|l|l|}
\hline 1 & 1 & 0 & 0 & 0 \\
\hline 1 & 1 & 0 & 0 & 0 \\
\hline 0 & 0 & 0 & 0 & 0 \\
\hline 0 & 1 & 1 & 1 & 0 \\
\hline
\end{tabular}

(a)

\begin{tabular}{|l|l|l|l|l|}
\hline 0.00 & 0.00 & 1.00 & 2.00 & 3.00 \\
\hline 0.00 & 0.00 & 1.00 & 2.00 & 3.00 \\
\hline 1.00 & 1.00 & 1.41 & 2.00 & 2.24 \\
\hline 1.41 & 1.00 & 1.00 & 1.00 & 1.41 \\
\hline 1.00 & 0.00 & 0.00 & 0.00 & 1.00 \\
\hline
\end{tabular}

(b)

Fig 4: (a) shows a binary image matrix, and (b) shows the corresponding distance transform.

\section{THE PROPOSED ALGORITHM}

This approach comes from the idea in geography. Relative several concepts: watershed, catchment basin, watershed ridge line, topological surface. The key of this method is to change the starting image into another image whose catchment basins are the objects or regions we want to identify. In the initial step one real life image of pears is chosen and read by the standard MAT LAB command "imread". This function reads a grayscale or color image from the file specified. In next step the original image is converted into gray scale using "rgb2gray" function. "rgb2gray" converts color images to grayscale by eliminating the hue and saturation information while retaining the luminance. The gray scale image is converted into a binary image using "im 2 bw". It converts the grayscale image to a binary image where the image is a combination of $o$ and 1 . This binary image is smoothed in the following step by using "imclose" followed by "imopen" function. The morphological open operation is an erosion followed by a dilation, and the morphological close operation is a dilation followed by an erosion, both using the same structuring element for both operations. These are and finally we sum up the watershed segmentation using distance transform using "bwdist" and "watershed" functions. Where "bwdist" computes the Euclidean distance transform of the binary image and "watershed" computes a label matrix identifying the watershed regions. The flowchart of the proposed method is given below in figure 5 . 


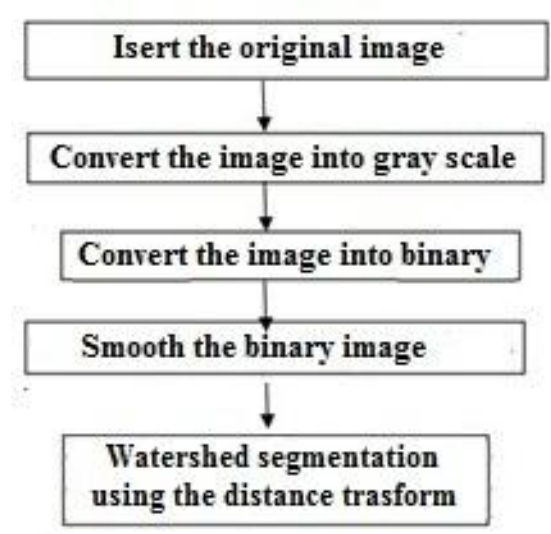

Fig 5: Flowchart of the proposed method.

\section{EXRERIMANTAL RESULTS}

In section 2, the watershed segmentation using distance transform technique is applied on the original image without smoothing the binary image. Some over segmentation is clearly visible there which makes difficulty for image understanding and analyzing of segmentation results (shown in figure 2). The concept of image smoothing is a useful solution for over segmentation. The proposed algorithm has been applied on the image of pears. Figure 6 to 10 shows the step wise output image which have been obtained by applying each process step of the algorithm one often another. It have been observed form figure 9, as obtained by the proposed algorithm that each and every segment of the final watershed segmented regions occupied an appreciable area and size and the number of such segmented regions are found to be much more less than which obtained previously in section 2 (figure 2 ). It has been further observed that numbers of regions are large. The histograms of the final resultant image by applying proposed method along with the histogram of segmented image after applying of watershed segmentation using distance transform without smoothing technique along have been shown in figures (10 and 11).

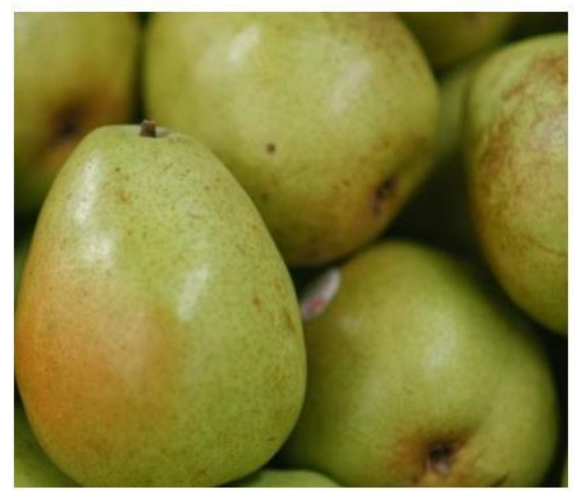

Fig 6: Original image.

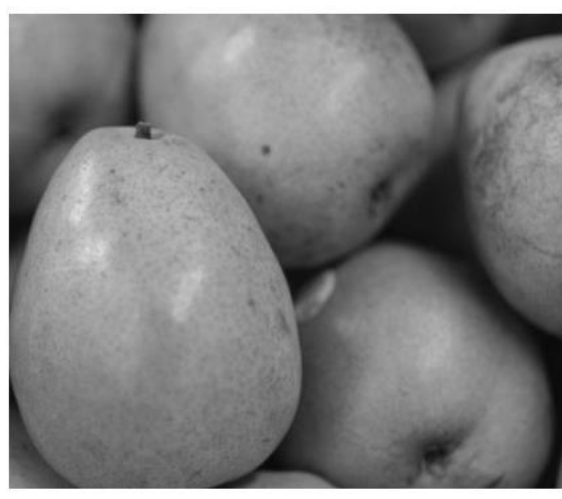

Fig 7: Gray scale image which obtained from the original image.

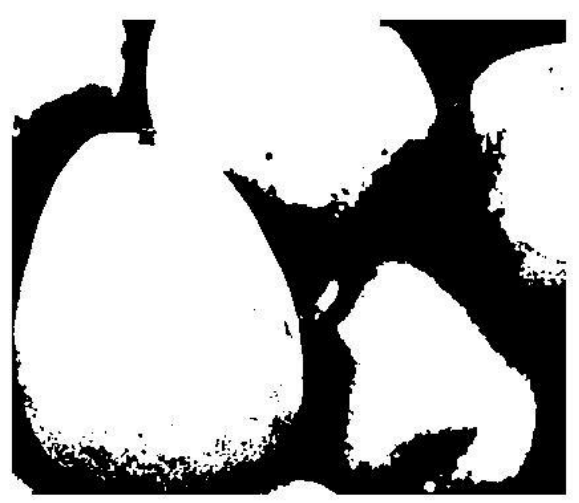

Fig 8: Binary image obtained from the gray scale image.

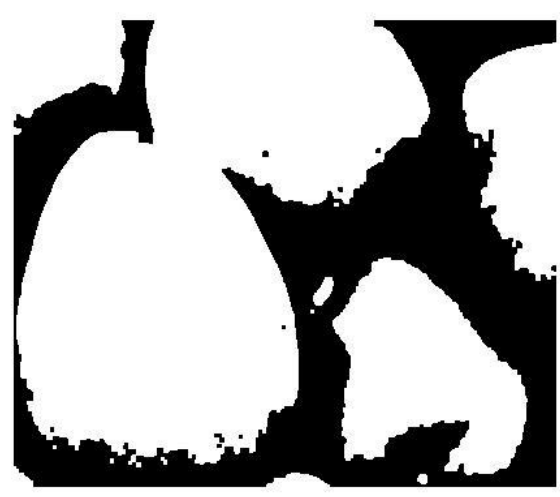

Fig 9: Binary image after smoothing. 


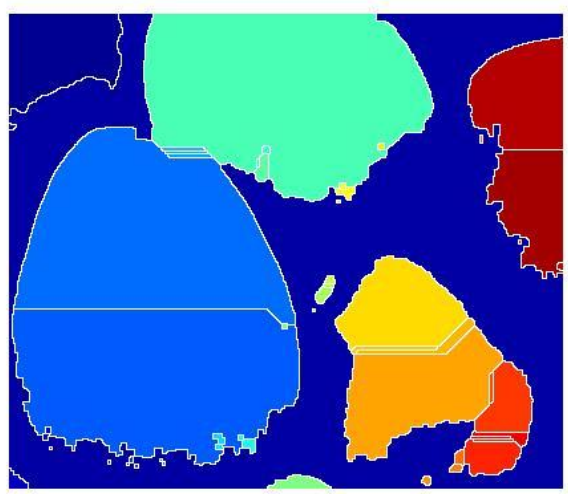

Fig 10: Segmented image after applying of watershed segmentation using distance transform with smoothing technique where the over segmentation is much lesser.

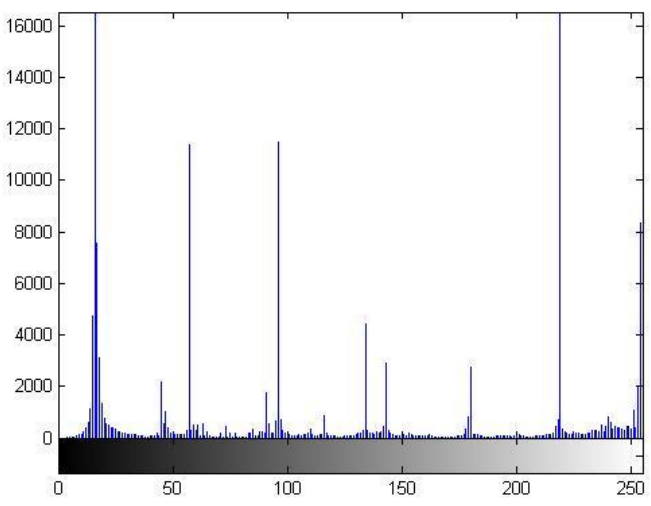

Fig 11: Histogram of segmented image after applying of watershed segmentation using distance transform without smoothing technique.

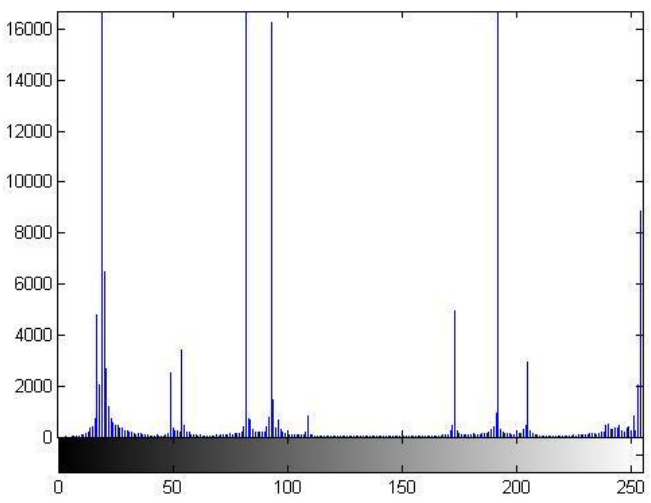

Fig 12: Histogram of segmented image after applying of watershed segmentation using distance transform with smoothing technique.

\section{CONCLUTSONS}

The present study has aimed at to provide an easy method of the segmentation of the segmentation based on mathematical morphology. This algorithm uses the concepts of distance transform and image smoothing as a pre requisite to determine the segments. The final resultant image obtained through watershed segmentation using distance transform without smoothing the binary image are found to be over segmented and it becomes subsequent computer handling very much difficult. The present method has been found to yield better output in term of image quality, clarity and sharpness with the avoidance of over segmentation.

\section{ACKNOWLEDGEMENT}

The authors acknowledge the constant inspiration and encouragement from Professor P. K. Bose, Director, National Institute of Technology, Agartala.

\section{DEDICATION}

One of the others (Dibeyendu Ghoshal) dedicates the entire study to the loveliest and loving memory of his only one and younger sister Kumari Sumita Ghoshal who herself was a gem of the scholars, a symbol of wisdom and art, peerless beauty and simplicity, unfathomable knowledge and generosity.

\section{REFERENCES}

[1] R. Besl, R. Jain, "Three dimensional object recognition," ACM Computing Surveys, Vol. 17, pp. 75-145, 1985.

[2] P. Suetnes, P.Fua and A. J. Hanson, "Computational strategies for object recognition," ACM Computing Surveys, Vol. 24, pp. 05-61, 1992.

[3] K. Hohne, H. Fuchs, S. Pizer, "3D imaging in medicine: Algorithms, systems, Applications", Berlin, Germany, Springer-Verlag, 1990.

[4] R. Besl, R. Jain, "Three dimensional object recognition," ACM Computing Surveys, Vol. 17, pp. 75-145, 1985.

[5] M. Kunt, M. Bernard and R. Leonardi, "Recent results in high compression image coding," IEEE Trans. on Circuits and Systems, Vol.34, pp.1306-1336, 1987.

[6] M. Bomans, K. Hohne, U. Tiede and M. Riemer, "3D segmentation of MR images of the head for 3D display," IEEE Transactions on Medical imaging, Vol.9, pp. 253277, 1990.

[7] F. Meyer, S. Beucher, "Morphological Segmentation", Journal of Visual Communication and Image Representation, 1, pp. 21-46, 1990.

[8] S. Beucher, M. Bilodeau, X. Yu. Road segmentation by watershed algorithms. Proceedings of the Pro-art vision group PROMETHEUS workshop, Sophia-Antipolis, France,pp pp. 299-314, April 1990.

[9] R. Harlick and L. Shapiro, "Image segmentation technique,” CVGIP, Vol.29, pp. 100-137, 1985.

[10] Vicent L. Solille P, Watershed in digital spaces, "An efficient algorithm based immersion simulations", IEEE Transections PAMI, pp. 538-598, 1991.M. Kunt, M. Bernard and R. Leonardi, "Recent results in high compression image coding," IEEE Trans. on Circuits and Systems, Vol.34, pp.1306-1336, 1987.

[11] F. D. Natale, G. Desoli, D. Glusto and G. Vernazza, "Polynomial approximation and vector quantization: A region based integration," IEEE transections on Communications, Vol.43, 1995.

[12] K. Haris, "A hybrid algorithm for the segmentation of 2D and 3D images," Master's thesis, University of Crete, 1994. 
[13] R. Harlick and L. Shapiro, "Image segmentation technique," CVGIP, Vol.29, pp. 100-137, 1985.

[14] S. Beucher, "Watershed, hierarchical segmentation and water fall algorithm," in Mathematical Morphology and Its Applications to Image Processing, Dordrecht, The Netherlands: Kluwer, 1994, pp. 69-76.

[15] Beucher, S., and Meyer, F. The morphological approach to segmentation: the watershed transformation. In Mathematical Morphology in Image Processing, E. R. Dougherty, Ed. Marcel Dekker, New York, ch. 12, pp. 433-481, 1993

[16] F. Meyer, S. Beucher, "Morphological Segmentation," Journal of Visual Communication and Image Representation,vol. 1, pp. 21-46, 1990.

[17] Gonzalez \& Woods, Digital Image Processing, 3rd edition, Prentice Hall India, 2008.

[18] K. Haris,"Hybrid image segmentation using watersheds and fast region merging," IEEE Trans Image Processing, 7(12), pp. 1684-1699, 1998.
[19] S. Thilagamani, N.Shanthi, "A Novel Recursive Clustering Algorithm for Image Oversegmentation", European Journal of Scientific Research, Vol.52, No.3, pp.430-436, 2011.

[20] Peter Eggleston, "Understanding Oversegmentation and Region Merging", Vision Systems Design, December 1, 1998.

[21] S. Beucher, "The Watershed Transformation Applied To Image Segmentation", Centre De Morphologie Mathématique. Ecole Des Mines De Paris, 1991.

[22] Shameem Akthar, D. RajyaLakshmi, Syed Abdul Sattar, "Suppression of Over Segmentation in Watershed Transform Using Pre Processing Method", International Journal of Computer Technology and Electronics Engineering, Volume 2, Issue 2, April 2012. 